\title{
Breast Cancer Risks and Risk Prediction Models
}

\author{
Christoph Engel $^{\mathrm{a}} \quad$ Christine Fischer ${ }^{\mathrm{b}}$ \\ a Institute for Medical Informatics, Statistics and Epidemiology, University of Leipzig, Germany \\ ${ }^{b}$ Institute of Human Genetics, University of Heidelberg, Germany
}

\section{Keywords}

Breast cancer - Ovarian cancer · Hereditary cancer risk . Risk prediction models

\section{Summary}

BRCA $1 / 2$ mutation carriers have a considerably increased risk to develop breast and ovarian cancer. The personalized clinical management of carriers and other at-risk individuals depends on precise knowledge of the cancer risks. In this report, we give an overview of the present literature on empirical cancer risks, and we describe risk prediction models that are currently used for individual risk assessment in clinical practice. Cancer risks show large variability between studies. Breast cancer risks are at $40-87 \%$ for BRCA 1 mutation carriers and $18-88 \%$ for BRCA2 mutation carriers. For ovarian cancer, the risk estimates are in the range of $22-65 \%$ for $B R C A 1$ and $10-35 \%$ for BRCA2. The contralateral breast cancer risk is high (10-year risk after first cancer $27 \%$ for BRCA1 and $19 \%$ for BRCA2). Risk prediction models have been proposed to provide more individualized risk prediction, using additional knowledge on family history, mode of inheritance of major genes, and other genetic and nongenetic risk factors. User-friendly software tools have been developed that serve as basis for decision-making in family counseling units. In conclusion, further assessment of cancer risks and model validation is needed, ideally based on prospective cohort studies. To obtain such data, clinical management of carriers and other at-risk individuals should always be accompanied by standardized scientific documentation.

\section{Introduction}

Women with pathogenic mutations in the BRCA1 and BRCA2 genes have a considerably increased risk to develop breast and ovarian cancer during their lifetime compared to the general population. The condition is characterized by incomplete penetrance, i.e. not all mutation carriers will develop cancer during their lifetime. Moreover, for those carriers who will develop cancer, the age of onset shows large interindividual heterogeneity between families and even within families. Families with BRCA1/2 mutations are characterized by multiple affected relatives and/or cases of early cancer onset. However, not in all families with a suspect cancer history can a BRCA1/2 mutation be found. Nonetheless, individuals from such $B R C A 1 / 2$-negative families are also considered to have an elevated cancer risk.

Clinical management strategies currently comprise intensified surveillance for early breast cancer detection using multimodal imaging techniques, or prophylactic surgery such as bilateral mastectomy or salpingo-oophorectomy. Genetic counseling of carriers and other at-risk individuals and personalized clinical decisionmaking highly depend on knowledge, as precise as possible, on the individual age-dependent cancer risks.

Since the discovery of the BRCA1 and BRCA2 genes in the middle of the 1990s, the breast and ovarian cancer risks for mutations carriers have been investigated in numerous studies [1-23]. The results from these studies serve as a major source of information for risk communication and tailored clinical decision-making. In addition, a variety of risk prediction models were developed in the past years in order to determine personalized cancer risks based on individual features such as familial cancer history, genetic test results, and also non-genetic risk factors. These models are particularly helpful for clinical decision-making in those families without a known pathogenic mutation despite strong evidence of a hereditary background.

In this paper, we aim to give an overview of the present knowledge on empirical breast and ovarian cancer risks that were investigated in epidemiological studies. Moreover, we present and describe different risk prediction models currently used for individual risk assessment in clinical practice.

\section{KARGER \\ Fax +497614520714

Christoph Engel, MD

Institute for Medical Informatics, Statistics and Epidemiology (IMISE)

University of Leipzig

Haertelstrasse 16-18, 04107 Leipzig, Germany

christoph.engel@imise.uni-leipzig.de 


\section{Cancer Risks of BRCA1/2 Mutation Carriers}

It has been demonstrated that the cancer risks in $B R C A 1 / 2 \mathrm{mu}$ tation carriers depend on the family history and also on the type of mutation [1, 13, 23-25]. Early epidemiological studies found breast cancer risks of up to $87 \%$ for $B R C A 1 / 2$ mutation carriers, whereas ovarian cancer risks were reported to be $64 \%$ for $B R C A 1$ and $27 \%$ for BRCA2 mutation carriers $[5,12,26]$. However, these cancer risk estimates were based on selected families with multiple cases and thus tended to overestimate the risks for unselected carriers or those with a less pronounced family history. Other studies that were based on less stringently selected families showed lower risks. Taken all studies together, the breast cancer penetrance is in the range of $40-87 \%$ for $B R C A 1$ mutation carriers and $18-88 \%$ for $B R C A 2$ mutation carriers. For ovarian cancer, the risk estimates are between $22-65 \%$ for $B R C A 1$ and $10-35 \%$ for $B R C A 2$. It is important to recognize that these studies are heterogeneous with regard to size, mode of ascertainment (population based vs. high-risk families), and the statistical methods of risk estimation. Consequently, there is considerable variation in the reported risks. In order to provide a comprehensive set of cancer risk estimates for BRCA mutations carriers that can be used by counselors and clinicians, Chen and Parmigiani [27] conducted a meta-analysis of 10 different studies based on strict selection criteria regarding study design and appropriate methodology. They report meta-analytic mean cumulative breast cancer risks at age 70 of $57 \%$ for $B R C A 1$ and $49 \%$ for BRCA2 mutation carriers (table 1). The ovarian cancer risks were $40 \%$ for $B R C A 1$ and $18 \%$ for $B R C A 2$ mutation carriers. Lifetime risks are a convenient measure to quantify and compare disease risks in different populations. However, in daily clinical practice and at the individual level, it is more appropriate to communicate cumulative cancer risks for a comprehensible time interval, e.g. 10 years, starting from the current age of the yet unaffected counselee. Therefore, in their meta-analysis, Chen and Parmigiani [27] also provided a tabulation of the remaining cancer risks. As an example, table 2 shows the 10 -year risks for breast and ovarian cancer for currently unaffected mutation carriers, depending on the current age of the counselee.

It is well known that $B R C A 1 / 2$ mutation carriers are also at an elevated risk of developing contralateral breast cancer (CBC) after a previous unilateral breast cancer. To date, a variety of studies have evaluated the risks of $\mathrm{CBC}$, most of which are retrospective in design [10, 22, 28-43]. A recent meta-analysis revealed CBC risks of $15 \%$ for $B R C A 1$ mutation carriers and $9 \%$ for BRCA2 mutation carriers 5 years after the first breast cancer [44]. The 10 -year risks were even higher with $27 \%$ and $19 \%$ for $B R C A 1$ and $B R C A 2$ mutation carriers, respectively (table 1 ). Moreover, a retrospective data analysis of the German Consortium for Hereditary Breast and Ovarian Cancer (GC-HBOC) has shown that younger age at first breast cancer was associated with a higher risk of CBC $[28,40]$.

Notably, most of the aforementioned studies are retrospective in design and require appropriate methods to adjust for the nonrandom selection of the study populations. The inherent problem of ascertainment bias can be overcome by conducting prospective
Table 1. Cumulative cancer risks from meta-analyses of penetrance studies $[27,44]$

\begin{tabular}{lll}
\hline & BRCA1, \% & BRCA2, \% \\
\hline Breast cancer (until age 70) & $57(47-66)$ & $49(40-57)$ \\
Ovarian cancer (until age 70) & $40(35-46)$ & $18(13-23)$ \\
CBC (within 10 years after first & $27(21-33)$ & $19(15-23)$ \\
$\quad$ breast cancer) & & \\
\hline
\end{tabular}

Table 2. The 10-year cumulative breast and ovarian cancer risks for currently unaffected BRCA mutation carriers by current age (adapted from [27])

\begin{tabular}{llllll}
\hline & \multicolumn{2}{l}{ Breast cancer, \% } & & \multicolumn{2}{l}{ Ovarian cancer, \% } \\
\cline { 2 - 3 } \cline { 5 - 6 } $\begin{array}{llllll}\text { Current } \\
\text { age, years }\end{array}$ & BRCA1 & BRCA2 & & BRCA1 & BRCA2 \\
\hline 20 & & & & \\
30 & $1.8(1.4-2.2)$ & $1(0.78-1.4)$ & & $1(0.68-1.8)$ & $0.19(0.09-0.47)$ \\
40 & $20(16.2-13)$ & $6.6(5.1-8.6)$ & $2.2(1.6-3.4)$ & $0.52(0.28-1)$ \\
50 & $22(18-27)$ & $15(12-19)$ & & $6.7(5.2-8.9)$ & $1.9(1.2-3.2)$ \\
60 & $19(15-24)$ & $17(14-22)$ & & $15(12-17)$ & $5.2(3.7-7.2)$ \\
\hline
\end{tabular}

cohort studies. However, prospective studies are comparably rare and limited in size and observation time. Recently, a larger prospective analysis of $1,887 \mathrm{BRCA1/2}$ mutation carriers from the EMBRACE (Epidemiological Study of Familial Breast Cancer) cohort study has been published [45]. The average cumulative breast cancer risks by age 70 for $B R C A 1$ mutation carriers were estimated to be $60 \%$ for breast cancer and $59 \%$ for ovarian cancer. For BRCA2 mutation carriers, the risks were $55 \%$ for breast cancer and $17 \%$ for ovarian cancer.

As mentioned above, the study design and population ascertainment may explain some of the observed cancer risk variation. However, there is growing evidence that the increased cancer risk of $B R C A 1 / 2$ mutation carriers can be modified by other genetic and non-genetic factors. In the past years, the international Consortium of Investigators of Modifiers of BRCA1/2 (CIMBA) has identified a number of single-nucleotide polymorphisms (SNPs) that are associated with breast cancer risk [46-53]. Although the association of each single SNP is small, their combination may have a considerable effect on cancer risks, with potential impact on clinical decision-making [50]. First results from the prospective EMBRACE study indicate that individual profiles of such genetic low-risk variants may allow clinically relevant risk stratification [45]. As regards non-genetic factors, a number of exposures and lifestyle factors such as reproductive history, hormone intake, smoking, and alcohol consumption have been considered to modify the cancer risk in $B R C A$ mutation carriers. However, the available evidence is still limited due the small sample sizes of the studies conducted so far [54].

Since the early 1990s, risk prediction models have been developed and extended to provide more personalized risk predictions. Most of the models are based on the empirical breast and ovarian cancer risk data from the aforementioned studies. Additionally, 
Table 3. Commonly used risk prediction models

\begin{tabular}{|c|c|c|c|c|c|c|c|c|}
\hline \multirow[t]{2}{*}{ Model } & \multicolumn{2}{|c|}{ Type of risk prediction } & \multicolumn{5}{|c|}{ Risk factors considered in the model } & \multirow[t]{2}{*}{ Web link } \\
\hline & $\begin{array}{l}\text { Remain- } \\
\text { ing } \\
\text { lifetime } \\
\text { risk }\end{array}$ & $\begin{array}{l}\text { Hetero- } \\
\text { zygote risk }\end{array}$ & Genetic & Non-genetic & $\begin{array}{l}\text { Tumor } \\
\text { pathology }\end{array}$ & $\begin{array}{l}B R C A 1 / 2 \\
\text { status }\end{array}$ & $\begin{array}{l}\text { Associated } \\
\text { tumors }\end{array}$ & \\
\hline Gail & $\mathrm{BC}$ & no & none & yes & yes & no & no & www.cancer.gov/bcrisktool \\
\hline eCLAUS & $\mathrm{BC}$ & yes & 1 gene & no & no & no & no & www.cyrillicsoftware.com \\
\hline BRCAPRO & $\mathrm{BC}, \mathrm{OC}$ & yes & BRCA1/2 & no & yes & yes & yes & $\begin{array}{l}\text { http://bcb.dfci.harvard.edu/ } \\
\text { bayesmendel/brcapro.php }\end{array}$ \\
\hline IBIS & $\mathrm{BC}, \mathrm{OC}$ & yes & $\begin{array}{l}\text { BRCA } 1 / 2+ \\
\text { single moderately } \\
\text { penetrant gene }\end{array}$ & yes & no & yes & yes & $\begin{array}{l}\text { www.ems-trials.org/ } \\
\text { riskevaluator }\end{array}$ \\
\hline BOADICEA & $\mathrm{BC}, \mathrm{OC}$ & yes & $\begin{array}{l}\text { BRCA } 1 / 2+ \\
\text { polygenic } \\
\text { component }\end{array}$ & no & yes & yes & yes & $\begin{array}{l}\text { http://ccge.medschl.cam. } \\
\text { ac.uk/boadicea/boadicea- } \\
\text { web-application }\end{array}$ \\
\hline
\end{tabular}

$\mathrm{BC}=$ Breast cancer, $\mathrm{OC}=$ ovarian cancer.

they consider breast and ovarian cancer status and age of onset for the counselee and relatives of any degree, the mode of inheritance of major genes, and other genetic and non-genetic risk factors.

\section{Breast Cancer Risk Prediction Models}

Breast and ovarian cancer risk prediction models have been developed to determine individual cancer risks for arbitrary women. Typically, clinicians apply these models to women from families with multiple affected family members and to early-onset breast and ovarian cancer patients. Table 3 shows the most commonly applied risk prediction models with their main features.

The Gail model uses current age, age at menarche, age at first life birth, number of previous breast biopsies, history of atypical hyperplasia, ethnicity, and number of affected first-degree female relatives for risk prediction [55]. No assumptions about genetic traits are made; ovarian cancer cases and extended family history are not taken into account. The Gail model is also not suitable for predicting cancer risks in $B R C A 1 / 2$ mutation carriers.

Other risk calculation models assume an underlying genetic model for breast cancer susceptibility and can fully utilize individual pedigree patterns. These so-called genetic risk prediction models include breast and ovarian cancer status and age of onset as well as the current age of healthy relatives of arbitrary degree. The model parameters are obtained from large epidemiological studies on age- and gene-specific empirical breast and ovarian cancer risks for mutation carriers as well as for the general population.

The earliest model, the Claus model, assumes 1 autosomal dominant gene with age-dependent penetrance [56]. It was developed before the BRCA1/2 genes had been identified and was later extended (eCLAUS model as implemented in the commercial pedigree drawing software CYRILLIC) to include ovarian cancer cases [57]. The Claus model does not take the BRCA1/2 status into account.
BRCAPRO is based on a model with the 2 genes BRCA1 and $B R C A 2$, and it can use BRCA1/2 screening results and family histories of $B R C A 1 / 2$-associated cancer types. BRCAPRO can account for multiple ethnicities, adjusts for mastectomies among relatives and, in its newest version, provides updated estimates of CBC penetrance [58].

BOADICEA (breast and ovarian analysis of disease incidence and carrier estimation algorithm) assumes that genetic susceptibility to breast cancer is due to $B R C A 1$ and $B R C A 2$ mutations and an additional polygenic component. It can use the mutation screening result, molecular tumor characteristics like estrogen receptor (ER), progesterone receptor (PR), human epidermal growth factor receptor 2 (HER2), and basal cytokeratin (CK) expression (CK5/6 and CK14), and family histories of BRCA1/2-associated cancers $[59,60]$.

IBIS assumes effects of BRCA1 and BRCA2 and models the residual genetic variability in terms of a third dominantly inherited common gene conferring moderate risks. IBIS can account for $B R C A 1 / 2$ screening results and additionally includes environmental factors such as parity, body mass index, age at menarche, age at menopause, age at first child birth, and benign breast diseases. IBIS can only handle healthy female probands, and the use of family history is restricted [60].

As a prerequisite for clinical use, breast cancer risk prediction models need to be externally validated and compared in prospective data sets. Only few such studies exist so far and they compare selected risk models based on different cohorts. Typically, calibration and discrimination of the models are investigated. Calibration compares how many breast cancer cases would have been expected by model prediction and how many cases have actually been observed within a defined time period, e.g. 10 years, starting from the current age of the counselee (observed-to-expected (O/E) ratio). Discrimination accuracy is a measure that describes how good the model can distinguish between women who are affected or not affected by breast cancer. A common descriptive measure for the discrimina- 
tive performance is the area under the curve (AUC) of the receiver operating characteristic (ROC). As a rule of thumb, clinical decision models should have an AUC of at least 0.7. Amir et al. [61] published a validation study based on 3,150 women comparing the models Gail, Claus, BRCAPRO, and IBIS. IBIS performed best, whereas the other models underestimated the breast cancer risk. The AUC varied between 0.72 and 0.76. Recently, Quante et al. [62] compared the Gail model with IBIS in a cohort of 1,857 women having an average breast cancer risk 3 times higher than in the general population. Regarding the 10-year breast cancer rates, IBIS showed better calibration and discrimination; the AUC was 0.7 in comparison to 0.63 for the GAIL model. In a small cohort of 358 mostly Ashkenazi high-risk women, both IBIS and BOADICEA overestimated the breast cancer risk. However, BOADICEA (O/E ratio $0.80,95 \%$ confidence interval (CI) $0.54-0.93$ ) was better calibrated than IBIS (O/E ratio $0.52,95 \%$ CI 0.32-0.87) [63]. In a large Australian cohort of 4,176 woman with European ancestry, BOADICEA was well calibrated (O/E ratio $0.92,95 \%$ CI $0.76-1.10)$ and showed good discrimination (AUC 0.7) [64]. In summary, non-genetic risk factors are included in Gail and IBIS; only IBIS and BOADICEA include BRCA1/2 and other genetic factors into the model.

The $\mathrm{CBC}$ risk can be calculated with eCLAUS, BOADICEA, and BRCAPRO. eCLAUS and BOADICEA make the simplifying assumption that the first and the second breast cancer are independently conditioned on $B R C A 1 / 2$ genotypes. However, evidence of a strong dependency of first and second breast cancer has recently been provided $[28,40,65]$. Thus, these risk prediction models are currently not suitable for $\mathrm{CBC}$ prediction and should not be used for this purpose until they have been adjusted and validated. A BRCAPRO upgrade has recently been released that explicitly accounts for the dependence between first and second breast cancer [58].

\section{Discussion}

Precise knowledge of individual breast and ovarian cancer risks is a major prerequisite for adequate counseling of at-risk individuals, in order to allow tailored decision-making regarding different clinical management options. Empirical data on the age-dependent risks obtained from epidemiological studies are not only important for risk communication. Such data is also important for risk calculation models as these models require valid cumulative breast cancer risks for $B R C A 1 / 2$ mutation carriers and for the general population.

The current international literature reveals large heterogeneity in the reported cancer risks for $B R C A 1 / 2$ mutation carriers between different studies. This might be partly due to different study designs and methods, but it may also reflect different characteristics of the study populations regarding other risk-modifying factors. Thus, the question arises as to which extent risk estimates from specific populations can be applied to other populations for risk prediction purposes. Moreover, this question of external validity must also be asked in the case of risk prediction models, since these are based on penetrance functions that were derived from specific populations. It therefore seems to be advisable to obtain risk estimates in the same populations and under the same conditions in which they are later used for risk prediction. Ideally, such risk estimation studies should be prospective in design, as retrospective data analyses are prone to different sources of bias, adjustment for which is often difficult and requires sophisticated statistical methods. In order to facilitate such data collections, individuals at an elevated risk of hereditary breast and ovarian cancer should be prospectively followed up in well-designed registries. In Germany, the GC-HBOC has established a large registry providing an important source of such data for high-risk families.

In contrast to empiric cancer risk estimates, risk prediction models offer the possibility to account for family history, known genes and their mode of inheritance, and several other genetic and non-genetic factors that modify the individual cancer risk. They are important tools to identify women at high risk of developing breast cancer and can be used to offer tailored prevention and clinical management. Work is still ongoing to improve these models by including additional risk factors, such as lifestyle factors or the large number of recently identified low-risk variants. This also includes calibration of model parameters to the specific target populations. Moreover, further prospective comparative validation studies are needed to evaluate whether the individual risk estimation is sufficiently precise for clinical implementation.

Typically, risk prediction models are used by choosing a cut-off value for the definition of 'high-risk' persons, who are then offered participation in intensified surveillance programs. Although several of the risk prediction models show good discrimination with AUCs $>0.7$, the consequences of a specific clinical decision based on a specific cut-off have to be carefully considered by evaluating the predictive values. For example, if a 3\% cut-off for the BOADICEA 10-year breast cancer risk were used to offer intensified surveillance in the Australian cohort as described in [64], the corresponding predictive values would be approximately $5 \%$ for the positive predictive value and approximately $99 \%$ for the negative predictive value. The cumulative 10 -year breast cancer rate in the total cohort was approximately $2.8 \%$. As a consequence, about $95 \%$ of all women under intensified surveillance would not get breast cancer in the next 10 years, and about $5 \%$ of the women may profit from early detection.

Therefore, we recommend communicating and discussing the predictive values and the clinical consequences of risk prediction using specific cut-off values. It might be a good strategy to use risk prediction models in selected subgroups with a higher 10-year breast cancer rate and therefore better predictive values. So far, the number of observed breast cancer cases in available cohorts is still too small to provide sufficient statistical power for the required subgroup analyses. Therefore, longer follow-up is needed in future validation studies.

\section{Disclosure Statement}

The authors have no conflicts of interests. 


\section{References}

1 Antoniou A, Pharoah PD, Narod S, et al.: Average risks of breast and ovarian cancer associated with BRCA1 or $B R C A 2$ mutations detected in case series unselected for family history: a combined analysis of 22 studies. Am J Hum Genet 2003;72:1117-1130.

2 Begg CB, Haile RW, Borg A, et al.: Variation of breast cancer risk among BRCA1/2 carriers. JAMA 2008;299: 194-201.

3 Brose MS, Rebbeck TR, Calzone KA, et al.: Cancer risk estimates for BRCA1 mutation carriers identified in a risk evaluation program. J Natl Cancer Inst 2002;94: 1365-1372.

4 Chen S, Iversen ES, Friebel T, et al.: Characterization of $B R C A 1$ and BRCA2 mutations in a large United States sample. J Clin Oncol 2006;24:863-871.

5 Easton DF, Ford D, Bishop DT: Breast and ovarian cancer incidence in BRCA1-mutation carriers. Breast Cancer Linkage Consortium. Am J Hum Genet 1995; 56:265-271.

6 Easton DF, Steele L, Fields P, et al.: Cancer risks in two large breast cancer families linked to BRCA2 on chromosome 13q12-13. Am J Hum Genet 1997;61:120-128.

7 Easton DF, Hopper JL, Thomas DC, et al.: Breast cancer risks for BRCA1/2 carriers. Science 2004;306:21872191; Author Reply 2187-2191.

$\checkmark 8$ Evans DG, Shenton A, Woodward E, et al.: Penetrance estimates for BRCA1 and BRCA2 based on genetic testing in a Clinical Cancer Genetics service setting: risks of breast/ovarian cancer quoted should reflect the can cer burden in the family. BMC Cancer 2008;8:155.

$\checkmark$ Fackenthal JD, Olopade OI: Breast cancer risk associated with BRCA1 and BRCA2 in diverse populations. Nat Rev Cancer 2007;7:937-948.

10 Ford D, Easton DF, Bishop DT, et al.: Risks of cancer in BRCA1-mutation carriers. Breast Cancer Linkage Consortium. Lancet 1994;343:692-695.

11 Ford D, Easton DF, Peto J: Estimates of the gene frequency of BRCA1 and its contribution to breast and ovarian cancer incidence. Am J Hum Genet 1995;57: 1457-1462.

12 Ford D, Easton DF, Stratton M, et al.: Genetic heterogeneity and penetrance analysis of the BRCA1 and $B R C A 2$ genes in breast cancer families. The Breast Cancer Linkage Consortium. Am J Hum Genet 1998; 62:676-689.

13 Gayther SA, Mangion J, Russell P, et al.: Variation of risks of breast and ovarian cancer associated with different germline mutations of the BRCA2 gene. Nat Genet 1997;15:103-105.

14 Gayther SA, Russell P, Harrington P, et al.: The contribution of germline BRCA1 and BRCA2 mutations to familial ovarian cancer: no evidence for other ovarian cancer-susceptibility genes. Am J Hum Genet 1999;65: 1021-1029.

15 Gilbert FJ, Warren RM, Kwan-Lim G, et al.: Cancers in $B R C A 1$ and $B R C A 2$ carriers and in women at high risk for breast cancer: MR imaging and mammographic features. Radiology 2009;252:358-368.

16 Hopper JL, Southey MC, Dite GS, et al.: Populationbased estimate of the average age-specific cumulative risk of breast cancer for a defined set of protein-truncating mutations in BRCA1 and BRCA2. Australian Breast Cancer Family Study. Cancer Epidemiol Biomarkers Prev 1999;8:741-747.

17 King MC, Wieand S, Hale K, et al.: Tamoxifen and breast cancer incidence among women with inherited mutations in BRCA1 and BRCA2: National Surgical Adjuvant Breast and Bowel Project (NSABP-P1) Breast Cancer Prevention Trial. JAMA 2001;286:2251-2256.

18 Satagopan JM, Offit K, Foulkes W, et al.: The lifetime risks of breast cancer in Ashkenazi Jewish carriers of $B R C A 1$ and BRCA2 mutations. Cancer Epidemiol Biomarkers Prev 2001;10:467-473.
Struewing JP, Hartge P, Wacholder S, et al.: The risk of cancer associated with specific mutations of BRCA1 and BRCA2 among Ashkenazi Jews. N Engl J Med 1997;336:1401-1408.

20 Warner E, Foulkes W, Goodwin P, et al.: Prevalence and penetrance of $B R C A 1$ and $B R C A 2$ gene mutations in unselected Ashkenazi Jewish women with breast cancer. J Natl Cancer Inst 1999;91:1241-1247.

21 Satagopan JM, Boyd J, Kauff ND, et al.: Ovarian cancer risk in Ashkenazi Jewish carriers of BRCA1 and BRCA2 mutations. Clin Cancer Res 2002;8:3776-3781.

22 van der Kolk DM, de Bock GH, Leegte BK, et al.: Penetrance of breast cancer, ovarian cancer and contralateral breast cancer in BRCA1 and BRCA2 families: high cancer incidence at older age. Breast Cancer Res Treat 2010;124:643-651

23 Metcalfe K, Lubinski J, Lynch HT, et al.: Family history of cancer and cancer risks in women with BRCA1 or BRCA2 mutations. J Natl Cancer Inst 2010;102:18741878.

24 Thompson D, Easton D: Variation in cancer risks, by mutation position, in BRCA2 mutation carriers. Am J Hum Genet 2001;68:410-419.

25 Thompson D, Easton D: Variation in BRCA1 cancer risks by mutation position. Cancer Epidemiol Biomarkers Prev 2002;11:329-336.

26 Easton DF, Bishop DT, Ford D, Crockford GP: Genetic linkage analysis in familial breast and ovarian cancer: results from 214 families. The Breast Cancer Linkage Consortium. Am J Hum Genet 1993;52:678-701.

27 Chen S, Parmigiani G: Meta-analysis of BRCA1 and BRCA2 penetrance. J Clin Oncol 2007;25:1329-1333.

28 Rhiem K, Engel C, Graeser M, et al.: The risk of contralateral breast cancer in patients from $B R C A 1 / 2$ negative high risk families as compared to patients from BRCA1 or BRCA2 positive families: a retrospective cohort study. Breast Cancer Res 2012;14:R156.

29 Metcalfe K, Gershman S, Lynch HT, et al.: Predictors of contralateral breast cancer in BRCA1 and BRCA2 mutation carriers. Br J Cancer 2011;104:1384-1392.

30 Metcalfe K, Lynch HT, Ghadirian P, et al.: Contralateral breast cancer in BRCA1 and BRCA2 mutation carriers. J Clin Oncol 2004;22:2328-2335.

31 Brekelmans CT, Tilanus-Linthorst MM, Seynaeve C, et al.: Tumour characteristics, survival and prognostic factors of hereditary breast cancer from BRCA2-, BRCA1and non-BRCA1/2 families as compared to sporadic breast cancer cases. Eur J Cancer 2007;43:867-876.

32 Pierce LJ, Levin AM, Rebbeck TR, et al.: Ten-year multi-institutional results of breast-conserving surgery and radiotherapy in BRCA1/2-associated stage I/II breast cancer. J Clin Oncol 2006;24:2437-2443.

33 Robson M, Svahn T, McCormick B, et al.: Appropriateness of breast-conserving treatment of breast carcinoma in women with germline mutations in $B R C A 1$ or BRCA2: a clinic-based series. Cancer 2005;103:44-51.

34 Robson ME, Chappuis PO, Satagopan J, et al.: A combined analysis of outcome following breast cancer: differences in survival based on BRCA1/BRCA2 mutation status and administration of adjuvant treatment. Breast Cancer Res 2004;6:R8-R17.

35 Haffty BG, Harrold E, Khan AJ, et al.: Outcome of conservatively managed early-onset breast cancer by BRCA1/2 status. Lancet 2002;359:1471-1477.

36 Verhoog LC, Brekelmans CT, Seynaeve C, et al.: Survival in hereditary breast cancer associated with germline mutations of BRCA2. J Clin Oncol 1999;17: 3396-3402.

37 Verhoog LC, Brekelmans CT, Seynaeve C, et al.: Contralateral breast cancer risk is influenced by the age at onset in BRCA1-associated breast cancer. $\mathrm{Br} \mathrm{J}$ Cancer 2000;83:384-386.
Breast Cancer Linkage Consortium: Cancer risks in BRCA2 mutation carriers. J Natl Cancer Inst 1999;91: 1310-1316.

39 Kirova YM, Stoppa-Lyonnet D, Savignoni A, Sigalet al.: Risk of breast cancer recurrence and contralateral breast cancer in relation to $B R C A 1$ and $B R C A 2$ mutation status following breast-conserving surgery and radiotherapy. Eur J Cancer 2005;41:2304-2311.

40 Graeser MK, Engel C, Rhiem K, et al.: Contralateral breast cancer risk in BRCA1 and BRCA2 mutation carriers. J Clin Oncol 2009;27:5887-5892.

41 Evans DG, Moran A, Hartley R, et al.: Long-term outcomes of breast cancer in women aged 30 years or younger, based on family history, pathology and BRCA1/BRCA2/TP53 status. Br J Cancer 2010;102: 1091-1098.

42 Malone KE, Begg CB, Haile RW, et al.: Populationbased study of the risk of second primary contralateral breast cancer associated with carrying a mutation in BRCA1 or BRCA2. J Clin Oncol 2010;28:2404-2410.

43 Garcia-Etienne CA, Barile M, Gentilini OD, et al.: Breast-conserving surgery in $B R C A 1 / 2$ mutation carriers: are we approaching an answer? Ann Surg Oncol 2009;16:3380-3387.

44 Molina-Montes E, Perez-Nevot B, Pollan M, et al.: Cumulative risk of second primary contralateral breast cancer in BRCA1/BRCA2 mutation carriers with a first breast cancer: a systematic review and meta-analysis. Breast 2014;23:721-742.

45 Mavaddat N, Peock S, Frost D, et al.: Cancer risks for $B R C A 1$ and BRCA2 mutation carriers: results from prospective analysis of EMBRACE. J Natl Cancer Inst 2013;105:812-822.

46 Antoniou AC, Sinilnikova OM, Simard J, et al.: RAD51 135G->C modifies breast cancer risk among BRCA2 mutation carriers: results from a combined analysis of 19 studies. Am J Hum Genet 2007;81:1186-1200.

47 Antoniou AC, Spurdle AB, Sinilnikova OM, et al.: Common breast cancer-predisposition alleles are associated with breast cancer risk in BRCA1 and BRCA2 mutation carriers. Am J Hum Genet 2008;82:937-948.

48 Antoniou AC, Sinilnikova OM, McGuffog L, et al. Common variants in LSP1, 2q35 and 8q24 and breast cancer risk for BRCA1 and BRCA2 mutation carriers. Hum Mol Genet 2009;18:4442-4456.

49 Antoniou AC, Wang X, Fredericksen ZS, et al.: A locus on $19 \mathrm{p} 13$ modifies risk of breast cancer in BRCA1 mutation carriers and is associated with hormone receptor-negative breast cancer in the general population. Nat Genet 2010;42:885-892.

50 Antoniou AC, Beesley J, McGuffog L, et al.: Common breast cancer susceptibility alleles and the risk of breast cancer for BRCA1 and BRCA2 mutation carriers: implications for risk prediction. Cancer Res 2010;70: 9742-9754.

51 Antoniou AC, Kuchenbaecker KB, Soucy P, et al. Common variants at $12 \mathrm{p} 11,12 \mathrm{q} 24,9 \mathrm{p} 21,9 \mathrm{q} 31.2$ and in ZNF365 are associated with breast cancer risk for $B R C A 1$ and/or BRCA2 mutation carriers. Breast Cancer Res 2012;14:R33.

52 Wang X, Pankratz VS, Fredericksen Z, et al.: Common variants associated with breast cancer in genome-wide association studies are modifiers of breast cancer risk in $B R C A 1$ and BRCA2 mutation carriers. Hum Mol Genet 2010;19:2886-2897.

53 Gaudet MM, Kirchhoff T, Green T, et al.: Common genetic variants and modification of penetrance of BRCA2 associated breast cancer. PLoS Genet 2010;6:e1001183.

54 Friebel TM, Domchek SM, Rebbeck TR: Modifiers of cancer risk in BRCA1 and BRCA2 mutation carriers: systematic review and meta-analysis. J Natl Cancer Inst 2014;106:dju091. 
55 Gail MH, Brinton LA, Byar DP, et al.: Projecting individualized probabilities of developing breast cancer for white females who are being examined annually. J Nat Cancer Inst 1989;81:1879-1886.

56 Claus EB, Risch N, Thompson WD: Autosomal dominant inheritance of early-onset breast cancer. Implications for risk prediction. Cancer 1994;73:643-651.

57 Narod SA, Goldgar D, Cannon-Albright L, et al.: Risk modifiers in carriers of BRCA1 mutations. Int J Cancer 1995;64:394-398.

58 Mazzola E, Chipman J, Cheng SC, Parmigiani G: Recent BRCAPRO upgrades significantly improve calibration. Cancer Epidemiol Biomarkers Prev 2014;23: 1689-1695.

59 Lee AJ, Cunningham AP, Kuchenbaecker KB, et al.: BOADICEA breast cancer risk prediction model: updates to cancer incidences, tumour pathology and web interface. Br J Cancer 2014;110:535-545.
60 Tyrer J, Duffy SW, Cuzick J: A breast cancer prediction model incorporating familial and personal risk factors. Stat Med 2004;23:1111-1130.

61 Amir E, Evans DG, Shenton A, et al.: Evaluation of breast cancer risk assessment packages in the family history evaluation and screening programme. J Med Genet 2003;40:807-814.

62 Quante AS, Whittemore AS, Shriver T, et al.: Breast cancer risk assessment across the risk continuum: genetic and nongenetic risk factors contributing to differential model performance. Breast Cancer Res 2012; 14:R144.
63 Laitman Y, Simeonov M, Keinan-Boker L, et al.: Breast cancer risk prediction accuracy in Jewish Israeli highrisk women using the BOADICEA and IBIS risk models. Genet Res (Camb) 2013;95:174-177.

64 MacInnis RJ, Bickerstaffe A, Apicella C, et al.: Prospective validation of the breast cancer risk prediction model BOADICEA and a batch-mode version BOADICEACentre. Br J Cancer 2013;109:1296-1301.

65 Rubino C, Arriagada R, Delaloge S, Le MG: Relation of risk of contralateral breast cancer to the interval since the first primary tumour. Br J Cancer 2010;102:213219. 